Sublexical Correspondences 1

Running head: SUBLEXICAL CORRESPONDENCES

Quantifying the reliance on different sublexical correspondences in German and English

Xenia Schmalz, Eva Marinus, Serje Robidoux, Sallyanne Palethorpe, Anne Castles \& Max Coltheart

ARC Centre of Excellence in Cognition and its Disorders

Department of Cognitive Science

Macquarie University

Sydney, Australia 


\begin{abstract}
The type of the sublexical correspondences employed during nonword reading has been a matter of considerable debate in the past decades of reading research. Nonwords may be read either via small units (graphemes), or large units (orthographic bodies). In addition, grapheme-to-phoneme correspondences may involve context-sensitive correspondences, such as pronouncing an "a" as / / when preceded by a "w". Here, we use an optimisation procedure to explore the reliance on these three types of correspondences in nonword reading. In Experiment 1, we use vowel length in German to show that all three sublexical correspondences are necessary and sufficient to predict the participants' responses. We then quantify the degree to which each correspondence is used. In Experiment 2, we present a similar analysis in English, which is a more complex orthographic system.
\end{abstract}

Keywords: Reading, Sublexical processing, Optimisation. 


\section{Quantifying the reliance on different sublexical correspondences in German and English}

How print is converted to speech is an important question, both from a theoretical and practical perspective. Sublexical translation processes have a central role in all current models of reading aloud (Coltheart, Rastle, Perry, Langdon, \& Ziegler, 2001; Perry, Ziegler, \& Zorzi, 2010; Plaut, McClelland, Seidenberg, \& Patterson, 1996; Seidenberg \& McClelland, 1989). The exact nature of this sound-to-speech conversion procedure, however, has been under considerable debate since the 1970s. In particular, the debate revolves around the question of whether this conversion relies predominantly on small units, such as graphemes, or larger units, such as orthographic bodies (e.g., "-ord") (Andrews, 1982; Coltheart et al., 2001; Cortese \& Simpson, 2000; Glushko, 1979; Jared, 2002) ${ }^{1}$. To a lesser extent, the literature has also drawn a distinction between context-sensitive and context-insensitive grapheme-to-phoneme correspondences (GPCs) and addressed the possibility that rather than relying purely on single-grapheme correspondences, in some cases the preceding or succeeding letters may provide a cue to the reader about the correct pronunciation of a grapheme (Perry, Ziegler, Braun, \& Zorzi, 2010; Treiman, Kessler, \& Bick, 2003; Treiman, Kessler, Zevin, Bick, \& Davis, 2006).

Thus, the literature reports three different types of correspondences that may be involved in sublexical decoding: context-insensitive GPCs, context-sensitive GPCs, and body-rime correspondences. Here, we propose a mathematical model based on an optimisation procedure that will allow us to fit the degree of reliance on each of the three types of correspondences. We begin with two experiments in German, where the language structure allows us to assess the independent contribution of each of the three types of correspondences. In two further experiments, we apply the same methodology 
to the English grapheme "a", which allows us to disentangle the reliance on context-sensitive GPCs compared to context-insensitive GPCs.

GPCs describe the relationship between graphemes and phonemes. The phoneme is the basic unit in spoken language, and a grapheme is the letter or letter cluster that corresponds to a single phoneme. The definitions of GPCs are straightforward in some cases; for example, the grapheme "b" always maps onto the phoneme /b/. This is an example of a context-insensitive GPC: regardless of the letters that precede or succeed the grapheme, its assigned phoneme does not change. However, this gets more complicated when we consider the GPC for a grapheme such as "a". In English, context-insensitive correspondences would dictate that "a" should be pronounced as in "cat". Using this correspondence, words like "was" and "false" would be considered irregular, meaning that the correct pronunciation is inconsistent with the GPC. Yet, upon closer inspection, the pronunciations of "was" and "false" are entirely predictable when the context of the grapheme "a" is taken into account: in "was", the "a" is preceded by a "w", which in most cases changes the pronunciation to / $/$, as in "wad" and "swan" 2 . This context-sensitive correspondence can be written as "[w]a" $\rightarrow / \mathrm{a} /$. The pronunciation of the vowel in "false" can be similarly predicted by a complex context-sensitive GPC, namely "[C]a[1][C]" $\rightarrow$ /o:/ (hereafter: a[1]-correspondence). It is worth noting that these context-sensitive correspondences are still GPCs, as they relate a single grapheme (in this case, "a") to the pronunciation of a single phoneme. Thus, GPCs can be subdivided into context-sensitive GPCs ("[w]a" $\rightarrow / \mathrm{\rho} /$ ) and context-insensitive GPCs ("a" $\rightarrow / \mathfrak{x} /$ ).

The concept of GPCs is important for the classical computational model of the dual-route framework, the DRC (Coltheart et al., 2001). This model has a sublexical route which converts print to speech via a set of GPCs that are explicitly specified. 
The sublexical route contains some context-sensitive correspondences $(\mathrm{N}=28$ - though the exact numbers vary according to the version of the DRC), but operates mostly on single-letter (e.g., "b" $\rightarrow / \mathrm{b} / ; \mathrm{N}=40$ ) and multi-letter (e.g., "th" $\rightarrow / \theta / ; \mathrm{N}=165$ ) context-insensitive GPCs.

There is also experimental evidence that stresses the importance of context-sensitive correspondences. One study reported the case of a patient with acquired surface dyslexia (Patterson \& Behrmann, 1997): since this patient could not correctly read irregular words like "colonel" and "yacht", it was thought that her lexical system was heavily damaged. However, not all irregular words were a problem: she was unimpaired with words that could be resolved by the context-sensitive "[w]a" $\rightarrow / \mathrm{\rho} /$ correspondence, such as "wad" or "swan". This demonstrates the presence of such a context-sensitive correspondence in the sublexical system. Furthermore, studies of nonword reading have shown that there is psychological reality to context-sensitive correspondences (Treiman et al., 2003, 2006): both adults and children tend to pronounce nonwords such as "twamp" with the vowel as in "swan", whereas control items such as "glamp" are pronounced via the context-insensitive GPC, $\rightarrow / æ /$. This further suggests that the context-insensitive correspondence "a" $\rightarrow / \mathfrak{x} /$ does not fully reflect the strategies used during nonword reading.

In addition to context-insensitive and context-sensitive GPCs, readers have been shown to rely on body-rime correspondences. Body-rime correspondences are the sublexical links between bodies and rimes, where bodies are defined as the vowel and optional final consonant(s) of a monosyllabic word (e.g., "-ark" in the word "bark"). The rime is the phonological equivalent to the orthographic body. A linguistic analysis has shown that bodies are a reliable predictor of vowel pronunciation in English (Treiman, Mullennix, Bijeljac-Babic, \& Richmond-Welty, 1995). 
Full reviews of the psychological reality of body-rime correspondences can be found elsewhere (Goswami \& Bryant, 1990; Ziegler \& Goswami, 2005). Most relevant in the current context are nonword reading studies addressing this issue, because these allow for a systematic exploration of the non-lexical correspondences that participants rely on when lexical information is not available. In English, nonwords can be created that would yield different responses depending on whether GPCs or body-rime correspondences are used. This is done by manipulating the regularity and consistency of the base word. A base word that conforms to the context-insensitive GPCs is said to be regular, while words that violate the correspondences are considered to be irregular. The concept of regularity only matters if reading occurs at least in part via GPCs. If nonlexical reading occurs only via body-rime correspondences, then the reliability (or lack thereof) of the GPC information should not influence reading at all; rather, only inconsistency of body-rime correspondences should affect reading latencies and accuracy (e.g., the two ways of pronouncing "-ave" in "have" and "save", see Ziegler, Stone, and Jacobs (1997)).

Nonword reading studies that aim to estimate the reliance on GPCs versus body-rime correspondences can use the regularity and consistency of a base word to generate nonwords that predict different responses depending on the types of correspondences that are used by the participant. Such studies are important, because nonword reading data can shed light on processing underlying sublexical information, while minimising confounds from lexical processing. Understanding this process has strong theoretical implications, because sublexical print-to-speech conversion mechanisms play an important role in all prominent models of reading.

In order to disentangle the different sublexical processes that take place during reading, the first step is to create nonwords for which different types of 
correspondences make different predictions. For example, from a regular and consistent word such as "fact", the onset can be changed to create a nonword, for example, "ract". In this case, both large and small correspondences make the same predictions for pronouncing this nonword. However, if we take an irregular, but consistent word, such as "talk", and change the onset to create the nonword "ralk", we can use the readers' pronunciations of this nonword to determine whether they relied on context-insensitive GPCs (in which case the item would be pronounced to rhyme with "talc") or body-rime correspondences (where it would rhyme with "talk"). Such studies have shown that GPCs cannot fully account for the types of pronunciations that participants give to such nonwords, but neither do body-rime correspondences (Andrews \& Scarratt, 1998; Brown \& Deavers, 1999; Perry, Ziegler, Braun, \& Zorzi, 2010; Pritchard, Coltheart, Palethorpe, \& Castles, 2012).

Thus, there is evidence for reliance on the three different types of print-to-speech correspondences, but there are still questions that remain to be answered. Firstly, previous studies do not distinguish between the reliance on context-sensitive GPCs and body-rime correspondences. For example, if a participant pronounces the nonword "palse" to rhyme with "false", it may be that a context-sensitive correspondence, "a[l]" $\rightarrow$ /o: / has been used to derive the pronunciation, rather than the body-rime correspondence that "-alse" $\rightarrow$ /orls/. As will be discussed later, this is a problem in the English language, as body-rime correspondences and context-sensitive correspondences are confounded.

Secondly, even though such studies can establish the psychological reality of certain types of correspondences, examining between-item differences cannot provide any estimation of the relative degree to which each type of correspondence plays a role. As previous literature has demonstrated the psychological reality of context-insensitive 
GPCs, context-sensitive GPCs, and body-rime correspondences, it is likely that all three correspondence types help the sublexical route to determine the pronunciation of a nonword. How such a conflict between different types of correspondences may be resolved by the cognitive system is addressed in detail in the General Discussion. The possibility of parallel activation of several sublexical correspondences raises the question of whether it is possible to quantify the degree to which each plays a role in determining the pronunciation of a novel word, which is a natural next step after demonstrating a sublexical correspondence's psychological reality. As discussed below, more sophisticated analyses are needed to estimate the relative importance of each type of correspondence.

In addition to establishing the psychological reality of different types of sublexical correspondences, a considerable body of research has explored cross-linguistic differences in the reliance on GPCs versus body-rime correspondences (Goswami, Gombert, \& De Barrera, 1998; Goswami, Porpodas, \& Wheelwright, 1997; Goswami, Ziegler, Dalton, \& Schneider, 2003; Ziegler, Perry, Jacobs, \& Braun, 2001; Ziegler, Perry, Ma-Wyatt, Ladner, \& Schulte-Körne, 2003). The psycholinguistic grain-size theory, a cross-linguistic theory of reading development and skilled reading, proposes that the degree of reliance on sublexical correspondences of different types varies across languages (Ziegler \& Goswami, 2005). In particular, the reliance on body-rime correspondences has been reported to be stronger in English than German (Ziegler et al., 2001, 2003). This is argued to be because in English, large units (i.e., bodies) are a better predictor of the pronunciation of a word than GPCs (Treiman et al., 1995): for a word like "calm", the pronunciation is inconsistent with the GPCs ("kælm") but can be derived from its body neighbours (palm, balm, etc.). In German, on the other hand, the GPCs are highly reliable, meaning that there are few exceptions 
to the correspondences (Ziegler, Perry, \& Coltheart, 2000), therefore smaller units are the preferred grain-size of German readers. In other words, there is a theoretical framework which predicts differences in the reliance on the units across languages. Therefore, it is desirable to develop a mathematical model quantifying the degree of reliance in different languages.

In summary, previous literature has shown reliance on three different types of correspondences in English: context-insensitive GPCs, context-sensitive GPCs, and body-rime correspondences. The psycholinguistic grain-size theory proposes that the reliance on the different types of correspondences differs across languages (Ziegler \& Goswami, 2005). In the present experiments, we introduce a new method of quantifying the reliance on each type of correspondence. In the first two experiments (1A and $1 \mathrm{~B})$, we used German nonwords to assess the degree of reliance on each type of correspondence. In Experiment 2A and 2B we extend the procedure to a more complex orthographic system, namely English.

\section{Experiment 1A}

The German language allows us to neatly assess the independent contributions of context-insensitive GPCs, context-sensitive GPCs, and body-rime correspondences in a nonword reading paradigm: It is possible to create a set of items which generate different predictions for vowel pronunciation, depending on which strategy is used.

In German, there is relatively little ambiguity in print-to-sound correspondences, compared to English. What little ambiguity there is stems mostly from vowel pronunciation (Ziegler et al., 2000): Each vowel can be pronounced as either long or short (e.g., "Schal" / Ja:l/ versus "Schall" / Jal/). In monosyllabic words, vowel length is often signalled by context. Some context-sensitive correspondences allow the reader to 
unambiguously determine vowel length; for example, any vowel followed by an " $h$ " is pronounced long ("V[h]" $\rightarrow$ long vowel). Other context-sensitive correspondences are less transparent. These correspondences are described by a German implementation of Coltheart et al.'s (2001) DRC (Ziegler et al., 2000). To allow the sublexical route to determine vowel length, it contains a set of context-sensitive super-rules: any vowel which is followed by only one consonant elicits a long vowel response (e.g., "Wal"), and a vowel which is followed by two or more consonants is pronounced short (e.g., "Wald"). These two rules can be summarised as follows: "V[C]" $\rightarrow$ long vowel, and $" \mathrm{~V}[\mathrm{C}][\mathrm{C}] " \rightarrow$ short vowel.

Although these super-rules capture the overall statistical distribution, there are also some exceptions, or words that would be irregular according to the German DRC (Ziegler et al., 2000). The word "Magd", for example, is pronounced with a long vowel; conversely the word "Bus" is pronounced with a short vowel. The presence of several bodies which consistently break the super-rules allows us to orthogonally manipulate the number of consonants in the body of a nonword, and the pronunciation of the base-words. Thus, we create a situation where the different types of correspondences (i.e., super-rules and body analogy) make different predictions about the pronunciation of the vowel.

For the present experiment, we can make a set of simple predictions if we assume that readers generally use only one type of correspondence: If only context-insensitive GPCs are used for German nonword reading, we expect that the likelihood of a short vowel pronunciation should be independent of any other orthographic features of the nonword. Such a GPC would predict many more short than long vowels, as the majority of vowels in German have the short pronunciation (Perry, Ziegler, Braun, \& Zorzi, 2010). If a context-sensitive super-rule is used, vowel length should be solely 
determined by the number of consonants following the vowel. In this case, even a nonword based on the irregular but consistent word such as "Magd" (e.g., "blagd") should be pronounced with a short vowel. These irregular-base-word items can distinguish between reliance on super-rules compared to body-rime correspondences: if body-rime correspondences are used, nonwords based on irregular consistent words should be pronounced to rhyme with their real-word counterparts.

\section{Methods}

Participants were 12 German native speakers who were staff or postgraduate students at Macquarie University, or members of the university's German society. As they lived in Australia, they were also fluent in English - a point which we will discuss in a later section. With one exception, all participants had completed secondary education in Germany and 10 had also attended German tertiary education. One participant had moved to Australia at the age of 5, but had attended a German-speaking school for 7 years.

The nonwords that were used for this experiment are listed in Appendix A. There were 30 nonwords in each of three conditions. The nonwords were created by changing the onsets of real words. All base-words were taken from a list of consistent German words (J. Ziegler, personal communication, 2012). The first condition used base-words with $\mathrm{V}[\mathrm{C}]$ bodies which were pronounced with a long vowel ("Jod" $\rightarrow$ "FOD"); the second condition was based on V[C][C] words with a short vowel ("Saft" $\rightarrow$ "BLAFT"). The third condition was derived from irregular words, which had either a $\mathrm{V}[\mathrm{C}]$ body but a short vowel ("mit" $\rightarrow$ "GIT") or a V[C][C] structure and a long vowel ("Jagd" $\rightarrow$ "BAGD"). The three conditions were matched on orthographic N (the number of real words that can be created by substituting one letter): $\mathrm{V}[\mathrm{C}]$ items 
had an average orthographic N-size of $1.73(\mathrm{SD}=1.46), \mathrm{V}[\mathrm{C}][\mathrm{C}]$ items had a mean of $2.10(\mathrm{SD}=1.69)$, and items with irregular base-words had a mean of $1.83(\mathrm{SD}=1.90)$. The mean body-N (number of real words with the same body) for the three conditions is $1.93(\mathrm{SD}=1.87), 2.40(\mathrm{SD}=1.98)$, and $1.37(\mathrm{SD}=1.00)$ respectively.

Participants were tested individually in a quiet room. Instructions were given in German by a native speaker. The participants were told that they would be asked to read nonwords which were created using German orthographic rules. The instructions emphasised that accuracy was more important than speed to discourage quick lexical processing, which might result in lexicalisation errors.

The items were presented using the DMDX software package (Forster \& Forster, 2003) in random order. Each trial consisted of a fixation cross, which remained in the centre of the screen for $500 \mathrm{~ms}$, followed by the item, which remained on the screen until the voice-key was triggered. Ten practice nonwords preceded the experiment. As all nouns in German are spelled with capital initial letters, presenting nonwords in all lower-case would provide an indication of word class of a nonword. Previous research has shown that information on the likely word class of a nonword affects its pronunciation (Campbell \& Besner, 1981). Therefore, all items were presented in upper case.

\section{Results}

Six trials $(0.6 \%)$ were excluded due to poor sound quality or premature voice-key triggering. The rest of the trials were scored by a German native speaker as pronounced with a long vowel, a short vowel, or incorrectly. For identifying incorrect responses, we used a lenient marking criterion: if a participant's response was consistent with a possible pronunciation of the GPCs, it was marked as correct (e.g., 
"spic" was marked as correct regardless of whether it was pronounced as /spik/ or as / $p$ pik/ - while, in German, "s" is typically pronounced as / $/$ / before "p" or "t", there are a few instances, such as loanwords, where it is assigned the pronunciation /s/).

Overall, $1.1 \%$ of all responses were classified as incorrect and excluded from subsequent analyses. Of primary interest were the proportions of long and short vowel responses and how they differed across condition. We split the Irregular-base-word condition by whether the bodies had one (hereafter referred to as V[C] Irregular; $\mathrm{N}=17$ ) or two $(\mathrm{V}[\mathrm{C}][\mathrm{C}]$ Irregular, $\mathrm{N}=13)$ consonants. Note that the two "irregular" conditions did not differ dramatically on any item characteristics: the mean number of letters was $3.88(\mathrm{SD}=0.34)$ and $4.36(\mathrm{SD}=0.63)$ for the $\mathrm{V}[\mathrm{C}]$ and $\mathrm{V}[\mathrm{C}][\mathrm{C}]$ conditions respectively, orthographic $\mathrm{N}$ was $1.88(\mathrm{SD}=2.19)$ and $1.79(\mathrm{SD}=1.58)$ respectively, and body- $\mathrm{N}$ was $1.56(\mathrm{SD}=1.15)$ and $1.29(\mathrm{SD}=0.61)$ respectively. The proportions of short vowel responses for each of the four item types (V[C] Regular, V[C][C] Regular, V[C] Irregular and V[C][C] Irregular) are listed in Table 1, along with the predictions according to each of the three types of correspondences.

[Table 1 about here]

In order to make the predictions more specific, we can use a corpus analysis to determine the percentage of times a vowel is pronounced as long or short under certain circumstances. For example, overall, $78.02 \%$ of all monosyllabic words are pronounced with a short vowel (Perry, Ziegler, Braun, \& Zorzi, 2010), therefore if German readers rely on context-insensitive GPCs, we expect them to give around the same percentage of short vowel responses. Among words with a single-consonant coda, $24.53 \%$ are pronounced with a short vowel, so we expect about the same percentage of short vowel responses to $\mathrm{V}[\mathrm{C}]$ nonwords, if only super-rules are used to determine vowel length. In Table 1, we present the predicted vowel lengths for each of the four conditions and by 
each of the three types of correspondences. For the context-insensitive GPCs and super-rules, these are calculated from the analyses presented in Perry, Ziegler, Braun, and Zorzi (2010). The predictions of the body-rime correspondences depend on the consistency ratio of the body. In the current study, we used only consistent items, where the body has only one pronunciation in real words. This means that if participants rely solely on body-rime correspondences, $100 \%$ of the pronunciations should be consistent with the base word vowel length.

The obtained percentages of long and short vowels (Table 1) are not consistent with the predictions of any one strategy we described above: vowel length responses are neither predominantly short in all four conditions, nor completely dependent on the number of consonants following the vowel, nor the vowel length of the base word. This is a clear indication that German readers rely on more than one type of correspondence for reading nonwords. Moreover, a closer look at Table 1 shows that no combination of two types of correspondences can account for the results, either: If context-insensitive GPCs and context-sensitive correspondences were the sole determiners of vowel length, we would not expect to find different proportions for the $\mathrm{V}[\mathrm{C}]$ Regular and $\mathrm{V}[\mathrm{C}]$ Irregular items - but we do. If context-insensitive GPCs and body-rime correspondences were the only predictors of vowel length, we would find no difference between the $\mathrm{V}[\mathrm{C}]$ Regular and the $\mathrm{V}[\mathrm{C}][\mathrm{C}]$ Regular items - and we do. If only context-sensitive correspondences and body-rime correspondences were used, we should observe less than $25 \%$ short vowel responses - which is not supported by the data.

\section{Modelling Vowel Pronunciations}

As shown above, it is not possible for a single or even a pair of types of correspondences to adequately fit the empirical data. It may be, however, that some 
combination of all three types of correspondences provides a good fit. Here we introduce a mathematical modelling approach that allows us to uncover more complex relationships between the types of correspondences. The goal is to weight the three strategies ${ }^{3}$ in a way that optimally fits the empirical data. More formally, we are seeking a set of $\beta$ weights that best satisfy the following mathematical model (one pair of equations for each item):

$$
\begin{gathered}
P_{i}(\text { Short })=\beta_{\text {gpc }} \times G P C_{\text {short }, i}+\beta_{\text {csc }} \times C S C_{\text {short }, i}+\beta_{\text {brc }} \times B R C_{\text {short }, i} \\
P_{i}(\text { Long })=\beta_{\text {gpc }} \times G P C_{\text {long }, i}+\beta_{\text {csc }} \times C S C_{\text {long }, i}+\beta_{\text {brc }} \times B R C_{\text {long }, i}
\end{gathered}
$$

where $G P C_{[l e n g t h], i}$ is the probability of item $i$ being pronounced with a vowel of the corresponding length according to the corpus analysis when using only context-insensitive (single-letter) GPCs as a predictor, $C S C_{[l e n g t h], i}$ is the probability according to context-sensitive super-rules, and $B R C_{[l e n g t h], i}$ is the probability according to the body-rime correspondence rules. Table 1 provides the average predictions for each condition, but the predictions from each correspondence were calculated separately for each item in the experiments. $P_{i}([$ length $])$ is the empirically observed proportion of the vowel length in Experiment 1A. ${ }^{4}$

At a first glance, this would appear to be a simple regression problem (with no intercept term). Linear regression would optimally select $\beta$ values that minimised the prediction error for (1) (indexed by the residual sum of squares). However, there are several reasons why this should not be thought of as regression. First, since the $\beta$ values are thought of as the degree to which a strategy applies in reading the items in Experiment 1, negative values would be uninterpretable. This means that all of our $\beta$ parameters must exceed 0 . This constraint can not be guaranteed by standard linear regression using ordinary least squares (Monfort, 1995).

Even with only positive $\beta$ s, there are two ways to interpret the weights. One 
could think of them as the contribution of each strategy to some sort of blending process that ultimately chooses the vowel pronunciation. In which case, we can simply fit the model in (1) above with the constraint that $\beta_{i} \geq 0, \forall i$. Alternately, one can think of the weights as the probabilities of adopting the vowel prediction from a given strategy. We prefer the latter interpretation (and discuss some evidence for it later), but it requires two further constraints: the $\beta$ weights must fall below 1 , and, since we assume the three strategies (GPCs, super-rules, and body-rime correspondences) are exhaustive, the three $\beta$ s must sum to 1 . The model can be formalised as:

$$
\begin{gathered}
P_{i}(\text { short })=\beta_{\text {gpc }} \times G P C_{\text {short }, i}+\beta_{c s c} \times C S C_{\text {short }, i}+\beta_{\text {brc }} \times B R C_{\text {short }, i} \\
P_{i}(\text { long })=\beta_{\text {gpc }} \times G P C_{\text {long }, i}+\beta_{c s c} \times C S C_{\text {long }, i}+\beta_{b r c} \times B R C_{\text {long }, i} \\
\text { where } \beta_{j} \in[0,1] \text { and } \Sigma \beta_{j}=1, \forall j \in\{g p c, c s c, b r c\}
\end{gathered}
$$

that is, we are seeking a set of probabilistic weights on the three strategies that minimises the prediction error of the model. The challenge here is to both efficiently search the available parameter space, and satisfy the $\Sigma \beta_{j}=1$ constraint. The first problem is a well-studied one in computer science and solutions are available that solve it. The second problem is largely solved by introducing an additional equation that can only be satisfied if $\Sigma \beta_{j}=1$, and giving that equation a strong influence on the final parameter set. The interested reader can find a fuller discussion of the implementation details in Appendix B.

Optimal weights in Experiment 1A. In Experiment 1A, we collected the proportion of short and long vowel responses to 90 items, and for each item we have the predicted probability of a short or long vowel pronunciation according to each of the three strategies. The strategy predictions were obtained from the corpus analysis undertaken by Perry, Ziegler, Braun, and Zorzi (2010). 
Using the technique described above, the native German readers in Experiment 1A appear to be relying most on GPCs $\left(\hat{\beta}_{g p c}=0.56\right)$, and to a lesser extent on super-rules $\left(\hat{\beta}_{c s c}=0.19\right)$, and body-rime correspondences $\left(\hat{\beta}_{b r c}=0.26\right)$. See Table 2 for a summary of the modelling results across all of the present experiments.

[Table 2 about here]

The above analysis contains a theoretically supported but strong assumption that readers use only the three strategies described in the introduction when reading nonwords. It is possible that other sources of information are used by German native speakers to determine vowel length. We can provide a simple test of this possibility by relaxing some of the constraints on the model, and observing how critical those constraints were to the optimisation results. To do this we removed the $\Sigma \beta_{j}=1$ constraint, and allowed the $\beta$ s to take on any positive weights in the fitting process. That is, we fit the following alternative model (some subscripts indicating length and item have been omitted for simplicity):

$$
P(\text { length })=\beta_{g p c} \times G P C+\beta_{c s c} \times C S C+\beta_{b r c} \times B R C, \text { where } \beta_{i}>0 \forall i
$$

If readers are adopting other strategies that are not well described by the GPC, super-rules and body-rime correspondence strategies, the incomplete nature of the model should be reflected in these alternate weights. The weights that optimise (3) were $\hat{\beta}_{g p c}=0.58, \hat{\beta}_{c s c}=0.14$, and $\hat{\beta}_{b r c}=0.24$. These values sum to 0.96 , suggesting that there is little need for a fourth strategy to describe the data. This does not conclusively rule out a role for any other strategies, but provides some evidence that the three strategies already tested are sufficient. That said, there is one additional strategy that could be playing a role: anti-body correspondences, or the probability of a vowel being pronounced as long or short based on the onset of the word. In this 
corpus of nonwords, the predictions from antibody-rime correspondences and context-insensitive GPCs are highly correlated, so it is difficult to disentangle the two strategies entirely, but it may be that anti-body rime correspondences are more important than context-insensitive GPCs and thus are a better predictor. To test whether or not anti-body correspondences were important for determining vowel pronunciations, we added a component to model (2):

$$
\begin{gathered}
P(\text { Length })=\beta_{g p c} \times G P C+\beta_{c s c} \times C S C+\beta_{b r c} \times B R C+\beta_{a b c} \times A B C \\
\text { where } \beta_{j} \in[0,1] \text { and } \Sigma \beta_{j}=1
\end{gathered}
$$

where the addition of $A B C$ represents the predictions from anti-body correspondences, and $\beta_{a b c}$ is the associated weight. Fitting (4) produced the same weights that resulted from (2) where the antibody-rime correspondences were not included. That is, $\hat{\beta}_{a b c}=0$, giving little reason to believe that any other strategies are being used in Experiment 1A.

Model fits. The optimisation procedure presented here is only useful if it arrives at a model that fits the data better than alternatives. To determine the effectiveness of the model, we calculated the correlation between the model predictions and the observed response patterns. For comparison, we did the same for the GPCs, context-sensitive correspondences (CSC), and body-rime correspondences (BRC) individually. As can be seen in Table 3, the optimisation process outperforms the other three alternatives in all four samples presented here. In experiment $1 \mathrm{~A}$, the correlation is .844 while the next best model (based on context-insensitive GPCs) correlates at .714 .

[Table 3 about here] 


\section{Discussion}

In Experiment 1A, we successfully used an optimisation procedure to quantify the degree of reliance on three types of sublexical correspondences: context-insensitive GPCs, context-sensitive GPCs, and body-rime correspondences. This can be achieved with the German language, because it is possible to create items where different correspondence types make different predictions about the vowel length pronunciation. Importantly, we found that all three types of correspondences are both necessary and sufficient to predict vowel length responses in a sample of German native speakers. Context-insensitive correspondences appear to be the strongest predictor. This is in line with the psycholinguistic grain size theory, which argues that the smallest unit size is favoured by readers of a language with predictable GPCs, such as German (Ziegler \& Goswami, 2005).

Experiment 1A has some limitations. It could be argued that the results are unreliable, firstly due to the small sample size and secondly because the participants were bilingual, and very fluent in English. It is unclear how fluency in English may affect the reliance on different types of correspondences in German. Even though we took care to only include German participants who learned to read and write in German from a young age, there is a possibility that their exposure to German reading material has been diminished by residing in an English-speaking country. It is also possible that their knowledge of English would change the preferred unit in their native language: for example, psycholinguistic grain size theory predicts that readers of English rely more heavily on larger grain sizes than readers of German (Ziegler \& Goswami, 2005), though it does not make any statements about sublexical processing in bilinguals. We address these concerns in Experiment 1B. 


\section{Experiment 1B}

In Experiment 1B we collected data with two different samples of German native speakers who live in Germany and are not exposed to English on an everyday basis. We hereafter refer to them as monolingual Germans, even though they are not strictly monolingual: due to globalisation, it would be difficult if not impossible to find Germans who have no knowledge of English. Having collected data with two different samples of monolingual Germans allows us to test the reliability of the modelling method described here. If our model arrives at similar weights for two independent samples from the same population, we can be more confident that our modelling procedure is stable and reliable.

\section{Methods}

The methods were almost identical to Experiment 1A. One item was replaced (due to a typo, the original item set contained an inconsistent item, "blen", which was changed to "blem" in Experiment 1B).

The first sample consisted of 10 German native speakers who were staff or students at the Freie Universität in Berlin. All had completed their schooling in Germany. The second sample consisted of 26 undergraduate students at Potsdam University. Again, all were native German speakers and had completed their education in Germany.

\section{Results}

The scoring procedure was identical to Experiment 1A. For the Berlin sample, there were two non-responses $(0.22 \%)$ and 15 errors $(1.67 \%)$. The Potsdam sample made $2.3 \%$ errors. A series of t-tests showed that the percentages of long and short 
vowel responses did not differ significantly for any of the conditions across the two samples, all $p>0.4$. Furthermore, fitting each sample separately using the model described in (2) produced very similar weights. For the participants from Berlin, the weights were $\hat{\beta}_{g p c}=0.40, \hat{\beta}_{c s c}=0.33$, and $\hat{\beta}_{b r c}=0.27$. For the participants from Potsdam they were $\hat{\beta}_{g p c}=0.37, \hat{\beta}_{c s c}=0.35$, and $\hat{\beta}_{b r c}=0.28$. This result is comforting, suggesting that the method introduced here is reliable across different samples from similar populations. Since there was little difference between the two samples, we collapsed across them yielding a sample of 36 native German monolinguals. Using this collapsed sample, our model produces $\hat{\beta}_{g p c}=0.38, \hat{\beta}_{c d c}=0.35$, and $\hat{\beta}_{b r c}=0.27$. As in Experiment 1A, the optimal parameter set outperforms the alternatives in fitting the observed data (Table 3).

German/English bilingual vs. German monolingual readers. Since Experiments $1 \mathrm{~A}$ and $1 \mathrm{~B}$ are based on the same set of items, we have the opportunity to compare how the bilingual readers differed from the monolingual readers. The critical question is whether or not the smaller $\hat{\beta}_{g p c}$ and larger $\hat{\beta}_{c s c}$ for monolinguals represents a real difference, or simply random variation. In the usual context of a linear regression model, this would be a simple matter of including the language status of the participants (bilingual vs. monolingual) in the model, and testing for an interaction between language status, and the GPC and/or CSC estimates. However, our modelling strategy violates many of the assumptions that allow for straightforward t-tests of the parameter estimates (given the constraints of our model, the parameter estimates are unlikely to be well-behaved, statistically). Instead we turn to a bootstrapping methodology to allow us to use the data to conduct non-parametric tests of the variability in our estimates.

To establish the reliability of the difference in the $\hat{\beta}_{g p c}$ and $\hat{\beta}_{c s c}$ estimates, we 
repeatedly resampled 90 items (with replacement) from the data set, and estimated the $\hat{\beta}_{i}$ f for both the bilingual and monolingual participants with each sample of items. Of 10,000 such samples, 9,890 (98.9\%) produced a larger GPC weight for the bilingual subjects than for the monolingual subjects (95\%CI of the difference: 0.019 to 0.327 ). Similarly, 9,634 (96.2\%) samples produced a larger CSC weight for the monolingual participants than for the bilingual participants (95\%CI: -0.011 to 0.317$)$. This suggests that the difference in the GPC weights is robust, while the difference in the CSC weights is slightly more tenuous. The difference in the BRC weights was not at all significant: $3,454(34.5 \%)$ of the samples produced larger BRC weights for bilinguals than for monolinguals (95\%CI: -.058 to .089). We also took advantage of these bootstrap samples to estimate the variability in the correlations from the optimal parameters in Table 3.

To summarise the results so far, the reliance on body-rime correspondences did not differ between monolingual and bilingual readers, but there was a very stable difference in the reliance on context-insensitive GPCs and a somewhat stable difference in the role of context-sensitive super-rules. Monolinguals relied less on context-insensitive GPCs and somewhat more on super-rules than bilinguals.

Individual differences. There is some ambiguity in interpreting the weights: as we collapsed across participants, the weightings do not give us any information about inter-individual participant variability. Theoretically, it is possible that all participants rely on the same strategies to the same extent, or that the weightings are reflective of the percentage of participants who rely on a particular strategy only. To address this, we generated the weighings for each individual participant in Experiments 1A and 1B. These are summarised in Figure 1. This figure shows that there is individual variability, but most participants rely on a combination of the three strategies. 
[Figure 1 about here]

Discussion

As in the previous experiment, we were able to quantify the degree of reliance on each of the three types of correspondences in two samples of monolingual German native speakers. Even though there is individual variation, we found, on average, almost identical reliance on the three strategies in two independent samples of German readers, suggesting that the procedure we introduced is reliable. The overall pattern of results was also broadly consistent with the findings from Experiment 1A, showing that reliance on all three types of correspondences is both necessary and sufficient to explain the vowel length pronunciations in German, and that context-insensitive correspondences are the major predictor of the vowel responses.

While the bilingual and monolingual participants' response patterns were similar, we did find some significant differences in terms of reliance on context-sensitive versus context-insensitive correspondences: bilingual participants show stronger reliance on context-insensitive correspondences and less reliance on context-sensitive correspondences. Two possible causes of the difference between German/English bilinguals and German monolinguals are the influence of English proficiency on reading in the bilingual sample, or a general difference in German reading proficiency. According to the psycholinguistic grain size theory, if the difference in weights is due to the influence of English (L2) on the choice of correspondences in German (L1), we would expect bilinguals to rely more on larger correspondences (context-sensitive correspondences or body-rime correspondences as opposed to context-insensitive correspondences). Developmental studies have shown that reliance on larger units differs as a function of reading efficiency, as younger children rely to a greater extent 
on context-sensitive rules (Treiman et al., 2006). In Experiment 1B, we found that bilingual participants rely more on context-insensitive rules, which is more in line with a proficiency explanation - bilinguals may be less proficient in reading German than monolinguals, as they are less exposed to German texts. As a result, they rely to a greater extent on the context-insensitive correspondences. ${ }^{5}$

\section{Experiment 2A}

The majority of prior research on the use of GPCs, context-sensitivity and body-rime correspondences has been conducted in English. In contrast to German, the English letter-to-sound correspondence system is highly complex, as a large set of correspondences on different levels are required to describe the relationship between print and speech (Venezky, 1970). In Experiment 2, we aimed to explore whether it is possible to apply the methodology which we introduced in Experiment 1 to quantify the degree of reliance on the same three strategies in a more complex system.

English, like German, contains some context-sensitive correspondences. However, there are no super-rules, or correspondences which apply to all vowels, as in German. Therefore, we concentrated solely on the grapheme "a", as its correct pronunciation can often be disambiguated by taking into account its context. By default, "a" is pronounced as in "cat" in Australian English, but there are several context-sensitive and multi-letter GPCs that can modify its pronunciation. The context-sensitive correspondence of interest here is the correspondence that an "a" preceded by a "qu" or " $\mathrm{w}$ " is pronounced as $/ \mathrm{\partial} /$. We chose this correspondence to assess reliance on context-sensitivity for two reasons: Firstly, previous research has shown that there is some psychological reality to this correspondence (Patterson \& Behrmann, 1997; Treiman et al., 2003). Secondly, unlike other context-sensitive GPCs (e.g., "a[l]" $\rightarrow$ 
/o:/), this correspondence is not confounded with body-rime analogy, as the modifier is located in the onset, before the vowel. This is therefore one of the few English context-sensitive correspondences that allows us to independently assess effects of context-sensitivity.

In order to create an item set equivalent to the German nonwords used in Experiment 1, we isolated English bodies with the vowel grapheme "a" which are consistently pronounced irregularly (Ziegler et al., 1997). There are five such bodies: "-alse", "-att", "-alk", "-alt", and "-ald". With one exception, they are confounded with the "a[l]" $\rightarrow$ /o:/ correspondence: the body "-att" only occurs in the word "watt" and therefore only has the / / /-pronunciation. As a result, and in contrast to the German experiment, the degree of reliance on body-rime correspondences cannot be assessed using this paradigm, because it is almost perfectly confounded with reliance on the "a[l]" context-sensitive correspondence.

In short, there are three possible pronunciations indicative of reliance on different types of correspondences. If English participants rely on context-insensitive GPCs, we should find that the majority of nonwords are pronounced with the /æ/-vowel. If context-sensitive correspondences are used, then in the conditions where a "qu" or "w" precedes the vowel we should find many /ə/-responses. If either body-rime correspondences or the "a[l]"-correspondence are used, the conditions with the consistently irregular bodies should be pronounced with an /o:/.

\section{Methods}

The participants were 19 undergraduate students at Macquarie University who were all native speakers of English.

We created four conditions of 18 words each (listed in Appendix A). All were 
monosyllables containing the single vowel grapheme "a". The first condition was created by taking consistently regular bodies (Ziegler et al., 1997) and adding an onset which does not change the pronunciation of the vowel (i.e., any onset that does not contain "w" or "qu"), resulting in nonwords like "hact" (this condition is hereafter referred to as $\mathrm{CS}+\mathrm{BR}+$, as both the context-sensitive correspondences, $\mathrm{CS}$, and the body-rime correspondences, BR, agree with the context-insensitive GPC "a" $\rightarrow / \mathfrak{x} /$ ). The second condition (CS+BR-, e.g., "halse") was based on bodies where the "a" is consistently pronounced as /o:/ (or / / for the body "-att"), and "normal" onsets, as in the first condition. Here, the body-rime correspondences predict an /o:/ pronunciation, and therefore disagree with the context-insensitive correspondence. The items in the third condition (CS-BR+, e.g., "wact") were based on regular bodies and onsets containing "w" or "qu", meaning that the context-sensitive "[qu,w]" a-correspondence contradicted the context-insensitive GPC while the body-rime correspondences did not. The fourth condition (CS-BR-, e.g., "qualse") had items with irregular bodies and onsets with "w" or "qu" - here both the context-sensitive correspondence and the body disagree with the context-insensitive GPC. As filler items, we used a set of unrelated nonwords.

The presentation was identical to Experiment 1, with items presented in random order and in upper case letters. As with Experiment 1, participants were instructed to read the items as accurately as possible, without putting them under time pressure.

Results

The results were scored by the fourth author (SP), a native Australian English speaker and an experienced transcriber, with the aid of spectral analysis using the EMU speech database System and associated speech analysis tools (Cassidy \& 
Harrington, 2001). SP was unaware of the aims of the experiment while she was transcribing the data. Unlike the German data, scoring the responses as correct or incorrect was more complicated. For the grapheme "a", there are at least five plausible pronunciations: as in "cat", as in "false", as in "what", as in "cake", and as in "car". We considered only the first three responses, as they were predicted either by the context-insensitive GPC, "a" $\rightarrow / æ /$, the context-sensitive GPC, "[qu,w]a" $\rightarrow / \mathrm{o} /$, or the body-rime correspondence "a[l]" $\rightarrow$ /o:/ context-sensitive correspondence. Other responses and errors made up $4.09 \%$ of the $\mathrm{CS}+\mathrm{BR}+$ condition, $24.85 \%$ of the CS+BR- condition, $6.43 \%$ of the CS-BR+ condition, and $20.76 \%$ of the CS-BRcondition, and were excluded from the subsequent analyses. The percentage of "other" responses is particularly high for the BR-conditions, partly because in English, a post-vocalic "l" creates ambiguity in the pronunciation of the vowel, such that a long /o:/ may become indistinguishable from the phoneme /ou/. The percentages of /æ/, /o:/ and / / responses are presented in Table 4, with the results from Experiment 2B for comparison.

[Table 4 about here]

\section{Modelling Vowel Pronunciations in English}

The modelling strategy for Experiment $2 \mathrm{~A}$ and $2 \mathrm{~B}$ required a small modification from that employed in Experiments 1A and 1B. In German, there are only two available vowel pronunciations for "a": short and long. In Australian English, there are three pronunciations available for items of Experiment 2. This means that we now 
592

need three equations per item:

$$
\begin{aligned}
P(æ) & =\beta_{g p c} \times G P C_{æ}+\beta_{c s c} \times C S C_{æ}+\beta_{b r c} \times B R C_{æ} \\
P(\jmath) & =\beta_{g p c} \times G P C_{\supset}+\beta_{c s c} \times C S C_{\supset}+\beta_{b r c} \times B R C_{\supset} \\
P(o:) & =\beta_{g p c} \times G P C_{o:}+\beta_{c s c} \times C S C_{o:}+\beta_{b r c} \times B R C_{o}: \\
& \text { where } \beta_{j} \in[0,1] \text { and } \Sigma \beta_{j}=1
\end{aligned}
$$

where each of the subscripted strategies indicates the likelihood of the subscripted pronunciation under that strategy; for example, $G P C_{æ}$ indicates the likelihood of an /æ/ response under the GPC strategy. The end result is a set of $\hat{\beta}_{i} \mathrm{~s}$ that fit all three pronunciations simultaneously.

The weightings are shown in Table 2 . The role of context-sensitive correspondences appears to be the most important in predicting the pronunciation of the grapheme "a", with, $\hat{\beta}_{c s c}=0.69$. Body-rime correspondences also appear to contribute significantly, $\hat{\beta}_{b r c}=0.26$, while the reliance on context-insensitive correspondences is very small, $\hat{\beta}_{g p c}=0.05$. Indeed, the bootstrapping procedure produced $\hat{\beta}_{g p c}=0$ in $43.3 \%$ of the samples, and $\hat{\beta}_{g p c}<0.1$ in $82.0 \%$, suggesting that the reliance on context-insensitive correspondences does not differ significantly from zero. Here again, the model is outperforming each of the independent strategies at predicting response patterns on an item by item basis (see Table 3), but when considering the model's ability to predict cell means (Table 4), it's clear this approach is less successful in English than it was in German.

\section{Discussion}

We quantified the reliance on different types of correspondences for English nonwords with the grapheme "a", using the same modelling technique we introduced in Experiment 1 for German, with some minor modifications. Although the results were 
less clear-cut than in German, we show that the procedure can be applied to a more complex orthography. The model fits in Table 4 indicate that the English orthography is not best suited for such an analysis. In particular, the poor model fits are due to many / / /-responses, even when these were not predicted by us. This may be a result of the complex phonology of English: the phonemes /o/ and /o:/ are very similar, therefore it is possible that the participants had a tendency to shorten /o:/-responses, which then became indistinguishable from the vowel $/ \mathrm{\partial} /$. The second possibility is that another source of information is used to determine vowel pronunciations in English which we did not take into account.

Despite these limitations, there are several conclusions that can be drawn from the results. Firstly, the weightings showed that in English the three strategies are neither necessary nor sufficient to predict the pronunciation of the grapheme "a". In contrast to German, we obtained a relatively high percentage of "other" responses for the English data, or pronunciations that were implausible according to any of the correspondences that we thought participants may use. Such a heterogeneity of nonword reading aloud responses has also been reported elsewhere (Andrews \& Scarratt, 1998; Pritchard et al., 2012). While this would be an interesting topic to pursue in further research, for our purposes we discarded the unusual pronunciations as we were interested in quantifying the reliance on the same three types of correspondences we showed to be critical to nonword reading in German. This high percentage of "other" responses shows that it is likely that other strategies, such as more complex context-sensitive correspondences or lexical analogy, are used during nonword reading in English. In other words, the three types of correspondences we described in the introduction are not sufficient to explain vowel responses to the grapheme "a" in English - which is in contrast to the findings we report for German. 
Secondly, a striking finding is that the context-insensitive correspondences are hardly used at all to derive the pronunciation of the grapheme "a". Rather, English readers rely heavily on the context-sensitive GPC, which can often be used to derive the correct pronunciation for English words.

These results imply that in the special case of the grapheme "a", it may not be necessary to rely on all three types of sublexical correspondences to explain the pattern of vowel responses. However, we consider it highly unlikely that context-insensitive GPCs are not used at all for reading in English. We relied solely on nonwords with the grapheme "a" to derive the weightings in Experiment 2, and its correct pronunciation can often be predicted by context. Arguably, this may falsely bias the weightings towards an apparent greater reliance on context-sensitive correspondences than we would observe if we used different graphemes for this procedure. However, we consider it likely that context-sensitivity plays an equally important role for other vowels in English: as is the case for the grapheme "a", vowel pronunciations in English are generally inconsistent, but can be often resolved context-sensitive correspondences (Treiman et al., 1995). Nonword reading studies have also provided evidence for the psychological reality of context-sensitive correspondences determining vowel pronunciation in English, other than the "[qu/w]a"-correspondence (Treiman et al., 2003, 2006). As described above, we focussed on the "[qu/w]a"-correspondence only because it is not confounded with body-rime correspondences - if we used any other context-sensitive correspondence we would be unable to distinguish it from reliance on body analogy.

However, we do stress that the almost exclusive reliance on context-sensitive correspondences in Experiment 2 is unlikely to generalise to the processing of more consistent graphemes in English, such as consonants. If, linguistically, 
context-insensitive correspondences are generally predictive of the correct

pronunciation, there is no pressure on the readers to take into account the surrounding letters for those particular graphemes.

As discussed in the introduction, the body-rime correspondences of English are confounded with context-sensitive correspondences. Instead of the German super-rules, we used an English context-sensitive correspondence that is not located in the body, namely the "[qu,w]a" $\rightarrow / \mathrm{\rho} /$ correspondence. However, we cannot fully disambiguate the reliance on body-rime correspondences and the "a[l]"-correspondence. Future studies using nonword reading should bear in mind that body-rime correspondences and context-sensitive correspondences are heavily confounded, and that an apparently irregular pronunciation of a nonword may show reliance on either context-sensitive correspondences or body-rime correspondences.

\section{Experiment 2B}

In Experiment 2B, we tested a sample of German/English bilingual speakers on the English item set. As with Experiment 1B, this will allow us to verify the weightings in a different sample, and explore potential differences between mono- and bilingual participants.

In Experiment 1, we argued that the differences that we found between the two samples are more consistent with an account based on reading proficiency rather than one based on the influence of acquiring a language with a deeper orthography.

However, it may be that an early acquired L1 shapes the cognitive system in a way that biases the processing of subsequently learnt languages towards familiar types of correspondences. If so, this would predict a difference between participants reading English nonwords depending on whether their first language was English (as in 
Experiment 2A) or German.

Methods

The participants were 13 native German speakers living in Australia

(undergraduate and graduate students at Macquarie University, academic staff, family and friends). Eight of them had also participated in Experiment 1A several months earlier, but did not know that the two studies were related. In this sample, all participants had lived in Germany for at least 18 years before moving to an English-speaking country. The items and procedure were identical to Experiment 2A. The participants were told that they would see English nonwords, and were asked to pronounce each item as if it were an English word that they are unfamiliar with.

Results

The same scoring system was used as for Experiment 2A. The proportions of /æ/, /ð/ and /o:/ responses for both Experiment 2A and 2B are presented in Table 4. German native speakers overall gave more "other" nonword responses, or vowel responses that were inconsistent with our predictors, compared to the English monolinguals in Experiment 2A: $15.74 \%, 23.61 \%, 17.95 \%$, and $8.80 \%$ for the CS+BR+, CS+BR-, CS-BR+ and CS-BR- conditions respectively.

We repeated the optimisation technique to derive strategy weights for this Experiment. Table 2 summarises the weights for each of the three strategies in Experiments 1A, 1B, 2A and 2B. The results of Experiment $2 \mathrm{~B}$ mirror the findings from Experiment 2A: Again, we find strongest reliance on context-sensitive correspondences, robust reliance on body-rime correspondences, and negligible reliance on context-insensitive correspondences. Numerically, the reliance on context-sensitive correspondences appear to be larger $\left(\hat{\beta}_{c s c}=0.61\right)$ than in the monolingual sample 
$\left(\hat{\beta}_{c d c}=0.69\right)$. Here again, the optimal parameters outperform the alternatives with a correlation of .717 (see Table 3).

Comparing bilingual to monolingual English readers. Using the same bootstrapping technique described in Experiment 1, we confirmed that the German-English bilingual participants relied more on body-rime correspondences (BRCs) than did the English monolinguals. In 9,998 (99.98\%) of the samples, $\hat{\beta}_{b r c}$ was larger for bilinguals than monolinguals (95\% CI of the difference: 0.046 to 0.150$)$. The two samples did not differ significantly in their reliance on context-insensitive (GPC) rules, but there is some evidence that the monolinguals may rely more on context-sensitive correspondences ( $91.72 \%$ of the samples, $95 \%$ CI: -0.039 to 0.160$)$.

\section{Discussion}

In Experiment 2B we collected data on English nonword pronunciation from German/English bilingual participants, which we then compared to the "a"-pronunciations of English monolinguals in Experiment 2A. Again, we find that the fits of the model are somewhat discrepant with the data, suggesting that the pronunciation of the letter "a" depends also on sources of information that are not included in our model. As in Experiment 2A, we found no reliance on context-insensitive GPCs in either group, and only a nonsignificant trend towards larger reliance on body-rime correspondences or the "a[l]" $\rightarrow$ /o:/ correspondence in English monolinguals than the German/English bilinguals.

We found broadly the same pattern among two different groups of participants; here, we once again demonstrate the reliability of the optimisation procedure. The significant difference in the reliance on body-rime correspondences suggest that German native speakers, even when they are highly proficient in English, continue to 
rely less on these large units than English monolingual participants. Thus, the native orthography appears to leave small but noticeable footprints in the cognitive processes underlying reading in a second language.

\section{General Discussion}

In four experiments, we explored the reliance on three different sublexical correspondence types in different populations. In Experiments $1 \mathrm{~A}$ and $1 \mathrm{~B}$, we found that German native speakers relied on all three strategies: the greatest weighting was found for context-insensitive GPCs, followed by context-sensitive GPCs (super-rules) and body-rime correspondences when reading German-derived nonwords. In Experiments $2 \mathrm{~A}$ and $2 \mathrm{~B}$, we applied the same procedure to quantify the types of correspondences that participants rely on to derive the pronunciation of the grapheme "a" in English. We found strong reliance on context-sensitive GPCs, some reliance on body-rime correspondences, and little evidence that context-insensitive GPCs play a large role in determining the pronunciation of the grapheme "a".

Cross-Linguistic Differences in the Choice of Sublexical Correspondences: Comparing Experiments 1 and 2

Previous theoretical work predicts cross-linguistic differences in the reliance on different units in German and English (Ziegler \& Goswami, 2005). Unfortunately, with the experiments in the current study it is impossible to make a direct quantitative comparison across the two languages as we are comparing two differently structured orthographic correspondences. An alternative approach is to conduct the analyses within the languages and point out the differences between them on a descriptive level.

Our data suggest that given a grapheme where context is very important in English (i.e., "a"), context-sensitivity becomes very important compared to German, 
where context-insensitive correspondences are the major predictor. This is true even for a situation where there are statistical regularities at the level of context-sensitive correspondences. This is broadly in line with the psycholinguistic grain size theory (Ziegler \& Goswami, 2005): as the context is often an important predictor of the correct pronunciation of English words, readers are forced to rely on larger units. Our data emphasises the importance of context-sensitive GPCs in an inconsistent orthography such as English. In German, on the other hand, context-insensitive correspondences are mostly sufficient to derive the correct pronunciation of an unfamiliar word, therefore this level of correspondences is preferred.

The reality of the cross-linguistic differences becomes more evident in a comparison of Experiments 1A and 2B. This is partly a within-subject design, and involves bilingual participants reading both the English and the German item sets. The differences between the weightings in these two experiments were remarkable, with the pattern of results being more similar to that of the monolinguals of the respective language. This shows that the language is the determining factor for the reliance on different unit sizes, rather than the language background of the participants.

From this comparison, we conclude that the language that a participant is asked to read in matters more than the participant's language background: comparing the participants in Experiments 1A and 2B shows that bilinguals rely on the three types of correspondences almost to the same extent as monolinguals do in their respective language. Thus, we conclude that the cross-linguistic differences in sublexical processing are language-specific: acquiring a deep versus shallow orthography from childhood does not shape the cognitive system, but rather encourages the reader to rely on certain types of correspondences above others in that particular orthography. Those preferences do not seem directly transferable to a later acquired orthography; 
instead, a reader develops a sensitivity to the most advantageous combination of strategies in the new language.

\section{Models of Reading}

The current study shows that both in English and in German, several correspondence types are used in parallel. There are multiple verbal models that postulate such a scenario (LaBerge \& Samuels, 1974; Patterson \& Morton, 1985; Taft, 1991, 1994; Ziegler \& Goswami, 2005). The theoretical contribution of the current paper is proposing a method to quantify the degree to which these are used, which can be used as a benchmark for computational models.

An open question then is whether the current computational models can simulate the obtained results. The parallel processing of various correspondences poses a computational problem: whenever there are conflicts between the pronunciations predicted by various correspondences, the system needs a way to resolve these. In English, this is important, because there are often cases where different sublexical correspondences provide conflicting information.

In Table 5, we provide the percentages of regular responses from two models which have been implemented both in English and in German, namely the DRC (Coltheart et al., 2001; Ziegler et al., 2000) and the CDP+ (Perry, Ziegler, \& Zorzi, 2007; Perry, Ziegler, Braun, \& Zorzi, 2010). For English, there is a newer version of the $\mathrm{CDP}+$, namely the CDP++ (Perry, Ziegler, \& Zorzi, 2010), which differs from the $\mathrm{CDP}+$ in several points: it has been trained on a larger word set, contains some parameter changes, and can also deal with polysyllabic words. We provide the simulation data from both versions of the model.

[Table 5 about here] 
Both the $\mathrm{CDP}+/ \mathrm{CDP}++$ and the $\mathrm{DRC}$ are dual route models of reading, where nonwords are read purely via a sublexical procedure. Therefore, the current data are relevant to both models, as it concerns the nature of sublexical processing. The distinguishing feature between the two models is the way in which this procedure operates. The DRC has a set of sublexical GPCs, which are manually programmed into the sublexical route. A GPC in the DRC is defined as the most frequent phoneme that co-occurs with a given grapheme. As described in the introduction, the DRC contains context-sensitive correspondences as well as single-letter and multi-letter correspondences, but there is some ambiguity when it comes to deciding which context-sensitive correspondences to include in the model. The current version of the English DRC does not contain either a "[w]a"- or an "a[1]"-correspondence, therefore it provides the response /æ/ to all items (see Table 5). For the second DRC simulation, we added some more context-sensitive correspondences, however this does not seem to reflect the overall responses given by participants, either, as it now underestimates the number of regular (i.e., /æ/) pronunciations given by the participants. For the German DRC, the GPCs that are used to determine vowel length are the super-rules (Ziegler et al., 2000). It is clear, both from the present study (see Table 5) and from Perry, Ziegler, Braun, and Zorzi (2010) that the super-rules are not sufficient to explain German nonword pronunciations.

The $\mathrm{CDP}+/ \mathrm{CDP}++$, like the $\mathrm{DRC}$, is grapheme-based, but it develops context-sensitivity because the grapheme-to-phoneme correspondences are derived via a learning algorithm, which uses real word knowledge to obtain the most likely correspondences between print and speech (Zorzi, 2010). Yet, the CDP + does not provide an optimal fit for either the German or the English data, as it often underestimates the number of regular pronunciations (see Table 5). In particular, the 
English $\mathrm{CDP}+$ and $\mathrm{CDP}++$ seem to take context-sensitive correspondences into account more than the participants do, as they underestimates the number of /æ/-responses for the CS- conditions. In German, the biggest discrepancy between the $\mathrm{CDP}+$ prediction and the behavioural data is in the BR-conditions, suggesting that $\mathrm{CDP}+$ does not develop the same degree of reliance on body-rime correspondences that participants do.

As neither of the computational models is compatible with the behavioural results, these data cannot be used to adjudicate between the DRC and CDP+ approach. (Note that this was not the aim of the study to begin with.) We therefore turn to verbal models to provide a theoretical framework that can explain our obtained results. One such model which provides a means for the cognitive system to resolve conflicts between different sublexical correspondences has been proposed by Taft (1991, 1994). This interactive activation model states that activation passes hierarchically from the smallest units, through subsyllabic and syllabic units and morphemes to whole words, which then gives access to the semantic concept. There are additional feedback connections, which send activation from larger to smaller units.

Taft's (1994) model also makes some explicit statements about cross-linguistic differences: the salient sublexical correspondences differ depending on the orthographic and phonological properties of the language. For example, while English readers parse words into orthographic-syllabic units called BOSSes (Taft, 1979, 1992), French readers rely more on the phonological syllable (Taft \& Radeau, 1995). In our experiments we found reliance on similar types of correspondences in English and German. Thus, the correspondences that have psychological reality in English and German appear to be very similar. It is noteworthy that English and German are very similar in terms of their phonological and orthographic structure, therefore we expect that the salient 
sublexical correspondences do not differ greatly. The situation might be different in other languages. For example, when there is a tendency for words to be polysyllabic and to contain fewer consonant clusters, as is the case in languages like Italian, Spanish, or Russian, body-rime correspondences are unlikely to play a large role in reading (Duncan et al., 2013; Kerek \& Niemi, 2012).

\section{Limitations and Future Directions}

The goal of the study was to identify an optimal combination of different sources of information in deciding which vowel pronunciation is most appropriate when there are two or more alternatives. A limitation of the model is that it makes no claims about the decision-making mechanisms that resolve the ambiguity, only that some sources of information are more influential than others. It may be that on each trial, the decision is based on a "winning strategy" in which case the weights represent the likelihood of a particular strategy winning. Alternately, it may be that all three sources of information are combined in a Bayesian sense of "what response is most likely correct given the mix of influences." In this case the model weights should be interpreted as the degree of influence that each strategy has on the decision process. The present study is not able to adjudicate between these alternatives (or any others that we may not have considered), so we refrain from making strong statements favouring one or the other. The extent to which nonword pronunciations remain stable in different situations, the factors that influence any variability, and the mechanisms that resolve ambiguity remain questions for future research. We do note, however that while there is considerably variability between subjects in terms of their strategy weights (see Figure 1), there is some recent evidence that readers can be grouped according to their choices (Robidoux \& Pritchard, 2014), so there may be more 
structure hiding within this variability.

A limitation of the paradigm as described in this paper is that it is better suited for across-subject comparisons than across-item comparisons, due to the small number of available items. This is a general problem with this approach: there are not many items where context-sensitive correspondences and body-rime correspondences can be dissociated, as these are intrinsically correlated. While it would be interesting to use the same paradigm for a different set of nonword or word items to explore systematic changes in the weightings associated with item characteristics such as frequency (for words) or word-likeness (as measured, e.g., by orthographic N), the small number of possible items prevents us from doing this in a meaningful way.

Arguably, the data reported in this paper are also limited by our focus on the grapheme "a" only. While this criticism applies to the English data, the German data can be generalised to predicting vowel length across different graphemes. The English results, and our conclusions based on these analyses, are therefore weaker than those from the German analyses. Nevertheless, understanding the principles underlying reading in languages other than English is essential for the long-term goal of describing all differences and similarities between reading in different languages, and thereby creating a universal model of reading (Frost et al., 2012). This is especially important given the focus of previous literature on English. English is considered to be an "outlier" orthography, therefore it is questionable to use it as a base for most models of skilled reading, reading development, and dyslexia (Share, 2008). While we acknowledge that, in the current context, the optimisation procedure works better for German than English, we argue that the English data provides a strong demonstration of the parallel use of different types of sublexical grain sizes, and in particular context-sensitive correspondences in English, new insights into cross-linguistic 
differences associated with the reliability of print-to-speech correspondences, and a new benchmark for computational models of reading aloud.

We believe that this approach also has some utility when applied to other areas of psycholinguistics. In future research, the same paradigm can be used to systematically explore the sources of individual differences that we report in the current study. The paradigm can also be used with children: previous literature has debated for decades whether children start learning to read using large or small units first (Goswami, 2002; Goswami \& Bryant, 1990; Hulme et al., 2002). Such explorations in group and individual differences are of theoretical and practical value. Future research can also apply the same mathematical procedure to any situation in which items can be created where different strategies yield different predictions. Other areas in psycholinguistics to which this paradigm can be extended could be topics such as stress assignment for polysyllabic words, because it has been shown that, in several languages, different cues are used by participants to determine the stress of a given nonword (Arciuli, Monaghan, \& Seva, 2010; Burani \& Arduino, 2004; Protopapas, Gerakaki, \& Alexandri, 2006; Ševa, Monaghan, \& Arciuli, 2009).

\section{Conclusions}

The current study contributes to the literature on cognitive processes underlying reading in several aspects. We show that context-insensitive GPCs, super-rules and body-rime correspondences are necessary and sufficient to explain the vowel length pronunciations in German; in English, context-insensitive GPCs play a smaller or negligible role in assigning the pronunciation of the grapheme "a". We introduce a method to quantify the degree of reliance on each of the three different sublexical correspondence types using statistical modelling. This technique can be used to test 
Sublexical Correspondences 42

929 other hypotheses by future studies. 


\section{References}

Andrews, S. (1982). Phonological recoding: Is the regularity effect consistent? Memory \& Cognition, 10(6), 565-575.

Andrews, S., \& Scarratt, D. R. (1998). Rule and analogy mechanisms in reading nonwords: Hough dou peapel rede gnew wirds? Journal of Experimental Psychology: Human Perception and Performance, 24(4), 1052.

Arciuli, J., Monaghan, P., \& Seva, N. (2010). Learning to assign lexical stress during reading aloud: Corpus, behavioral, and computational investigations. Journal of Memory and Language, 63(2), 180-196.

Brown, G. D., \& Deavers, R. P. (1999). Units of analysis in nonword reading: Evidence from children and adults. Journal of Experimental Child Psychology, $73(3), 208-242$.

Burani, C., \& Arduino, L. S. (2004). Stress regularity or consistency? Reading aloud Italian polysyllables with different stress patterns. Brain and Language, 90(1), $318-325$.

Byrd, R. H., Lu, P., Nocedal, J., \& Zhu, C. (1995). A limited memory algorithm for bound constrained optimization. SIAM Journal on Scientific Computing, 16(5), $1190-1208$.

Campbell, R., \& Besner, D. (1981). This and THAP: constraints on the pronunciation of new, written words. The Quarterly Journal of Experimental Psychology, 33(4), 375-396.

Cassidy, S., \& Harrington, J. (2001). Multi-level annotation in the Emu speech database management system. Speech Communication, 33(1), 61-77.

Coltheart, M., Rastle, K., Perry, C., Langdon, R., \& Ziegler, J. (2001). DRC: A dual route cascaded model of visual word recognition and reading aloud. Psychological 
Review, 108(1), 204.

Cortese, M. J., \& Simpson, G. B. (2000). Regularity effects in word naming: What are they? Memory \& Cognition, 28(8), 1269-1276.

Cox, F., \& Palethorpe, S. (2007). Australian English. Journal of the International Phonetic Association, 37(03), 341-350.

Duncan, L. G., Castro, S. L., Defior, S., Seymour, P. H., Baillie, S., Leybaert, J., et al. (2013). Phonological development in relation to native language and literacy: Variations on a theme in six alphabetic orthographies. Cognition, 127(3), $398-419$.

Forster, K. I., \& Forster, J. C. (2003). DMDX: A Windows display program with millisecond accuracy. Behavior Research Methods, 35(1), 116-124.

Frost, R., Behme, C., Beveridge, M. E., Bak, T. H., Bowers, J. S., Coltheart, M., et al. (2012). Towards a universal model of reading. Behavioral and Brain Sciences, $35(5), 263$.

Glushko, R. J. (1979). The organization and activation of orthographic knowledge in reading aloud. Journal of Experimental Psychology: Human Perception and Performance, 5(4), 674.

Goswami, U. (2002). In the beginning was the rhyme? A reflection on Hulme, Hatcher, Nation, Brown, Adams, and Stuart (2002). Journal of Experimental Child Psychology, 82(1), 47-57.

Goswami, U., \& Bryant, P. (1990). Phonological skills and learning to read. London: Wiley Online Library.

Goswami, U., Gombert, J. E., \& De Barrera, L. F. (1998). Children's orthographic representations and linguistic transparency: Nonsense word reading in English, French, and Spanish. Applied Psycholinguistics, 19, 19-52. 
Goswami, U., Porpodas, C., \& Wheelwright, S. (1997). Childrens orthographic representations in English and Greek. European Journal of Psychology of Education, 12(3), 273-292.

Goswami, U., Ziegler, J. C., Dalton, L., \& Schneider, W. (2003). Nonword reading across orthographies: How flexible is the choice of reading units? Applied Psycholinguistics, 24(2), 235-247.

Grömping, U. (2010). Inference with linear equality and inequality constraints using R: The package ic.infer. Journal of Statistical Software. (Forthcoming)

Hulme, C., Hatcher, P. J., Nation, K., Brown, A., Adams, J., \& Stuart, G. (2002). Phoneme awareness is a better predictor of early reading skill than onset-rime awareness. Journal of Experimental Child Psychology, 82(1), 2-28.

Jared, D. (2002). Spelling-sound consistency and regularity effects in word naming. Journal of Memory and Language, 46(4), 723-750.

Kerek, E., \& Niemi, P. (2012). Grain-size units of phonological awareness among Russian first graders. Written Language \& Literacy, 15(1), 80-113.

LaBerge, D., \& Samuels, S. J. (1974). Toward a theory of automatic information processing in reading. Cognitive Psychology, 6(2), 293-323.

Monfort, A. (1995). Statistics and econometric models (Vol. 2). Cambridge, UK: Cambridge University Press.

Patterson, K., \& Behrmann, M. (1997). Frequency and consistency effects in a pure surface dyslexic patient. Journal of Experimental Psychology: Human Perception and Performance, $23(4), 1217$.

Patterson, K., \& Morton, J. (1985). From orthography to phonology: A new attempt at an old interpretation. In K. Patterson, J. Morton, \& M. Coltheart (Eds.), Surface dyslexia (pp. 1217-1231). Hillsdale, NJ: Erlbaum. 
Perry, C., Ziegler, J. C., Braun, M., \& Zorzi, M. (2010). Rules versus statistics in reading aloud: New evidence on an old debate. European Journal of Cognitive Psychology, 22(5), 798-812.

Perry, C., Ziegler, J. C., \& Zorzi, M. (2007). Nested incremental modeling in the development of computational theories: the $\mathrm{CDP}+$ model of reading aloud. Psychological Review, 114(2), 273.

Perry, C., Ziegler, J. C., \& Zorzi, M. (2010). Beyond single syllables: Large-scale modeling of reading aloud with the connectionist dual process $(\mathrm{CDP}++)$ model. Cognitive Psychology, 61(2), 106-151.

Plaut, D. C., McClelland, J. L., Seidenberg, M. S., \& Patterson, K. (1996).

Understanding normal and impaired word reading: computational principles in quasi-regular domains. Psychological Review, $103(1), 56$.

Pritchard, S. C., Coltheart, M., Palethorpe, S., \& Castles, A. (2012). Nonword reading: Comparing dual-route cascaded and connectionist dual-process models with human data. Journal of Experimental Psychology: Human Perception and Performance, 38(5), 1268-1288.

Protopapas, A., Gerakaki, S., \& Alexandri, S. (2006). Lexical and default stress assignment in reading Greek. Journal of Research in Reading, 29(4), 418-432.

R Core Team. (2013). R: A language and environment for statistical computing [Computer software manual]. Vienna, Austria. Available from http://www.R-project.org/

Robidoux, S., \& Pritchard, S. C. (2014). Hierarchical clustering analysis of reading aloud data: a new technique for evaluating the performance of computational models. Frontiers in Psychology, 5.

Seidenberg, M. S., \& McClelland, J. L. (1989). A distributed, developmental model of 
word recognition and naming. Psychological Review, 96(4), 523.

Ševa, N., Monaghan, P., \& Arciuli, J. (2009). Stressing what is important:

Orthographic cues and lexical stress assignment. Journal of Neurolinguistics, 22(3), 237-249.

Share, D. L. (2008). On the anglocentricities of current reading research and practice: the perils of overreliance on an" outlier" orthography. Psychological Bulletin, $134(4), 584$.

Taft, M. (1979). Lexical access-via an orthographic code: The basic orthographic syllabic structure (BOSS). Journal of Verbal Learning and Verbal Behavior, $18(1), 21-39$.

Taft, M. (1991). Reading and the mental lexicon. Hillsdale, NJ: Psychology Press.

Taft, M. (1992). The body of the BOSS: Subsyllabic units in the lexical processing of polysyllabic words. Journal of Experimental Psychology: Human Perception and Performance, 18(4), 1004.

Taft, M. (1994). Interactive-activation as a framework for understanding morphological processing. Language and Cognitive Processes, 9(3), 271-294.

Taft, M., \& Radeau, M. (1995). The influence of the phonological characteristics of a language on the functional units of reading: A study in French. Canadian Journal of Experimental Psychology/Revue canadienne de psychologie expérimentale, $49(3), 330$.

Treiman, R., Kessler, B., \& Bick, S. (2003). Influence of consonantal context on the pronunciation of vowels: A comparison of human readers and computational models. Cognition, 88(1), 49-78.

Treiman, R., Kessler, B., Zevin, J. D., Bick, S., \& Davis, M. (2006). Influence of consonantal context on the reading of vowels: Evidence from children. Journal of 
Experimental Child Psychology, 93(1), 1-24.

Treiman, R., Mullennix, J., Bijeljac-Babic, R., \& Richmond-Welty, E. D. (1995). The special role of rimes in the description, use, and acquisition of English orthography. Journal of Experimental Psychology: General, 124(2), 107.

Venezky, R. L. (1970). The structure of English orthography (Vol. 82). The Hague: Mouton.

Ziegler, J. C., \& Goswami, U. (2005). Reading acquisition, developmental dyslexia, and skilled reading across languages: a psycholinguistic grain size theory. Psychological Bulletin, 131(1), 3.

Ziegler, J. C., Perry, C., \& Coltheart, M. (2000). The DRC model of visual word recognition and reading aloud: An extension to German. European Journal of Cognitive Psychology, 12(3), 413-430.

Ziegler, J. C., Perry, C., Jacobs, A. M., \& Braun, M. (2001). Identical words are read differently in different languages. Psychological Science, 12(5), 379-384.

Ziegler, J. C., Perry, C., Ma-Wyatt, A., Ladner, D., \& Schulte-Körne, G. (2003). Developmental dyslexia in different languages: Language-specific or universal? Journal of Experimental Child Psychology, 86(3), 169-193.

Ziegler, J. C., Stone, G. O., \& Jacobs, A. M. (1997). What is the pronunciation for -ough and the spelling for $/ \mathrm{u} /$ ? a database for computing feedforward and feedback consistency in English. Behavior Research Methods, 29(4), 600-618.

Zorzi, M. (2010). The connectionist dual process (CDP) approach to modelling reading aloud. European Journal of Cognitive Psychology, 22(5), 836-860. 


\section{Appendix A: German and English nonwords used in}

Experiments 1 and 2

\section{German}

$V[C]$ Reg. blaf blen (blem in Exp. 1B) blod breg brel brul flom flüb fryp grät grem grom grul klid klur knul krel kril krön pflyp pid plät plön prod schmün schraf schwüb speg zwül zwun

$V[C][C]$ Reg. bamt birt blaft bling boft brals chrolf falb flarg flerk gärm ginn gralb gunt kall kaxt kerv kluns knell pals peld pfern pulk purf schern spalf stelf sturg zeng zwurt

$V[C]$ Irreg. bax blex blig bres flim flis git glef glip krex krin krip pfis spic stef zwix zwok

$V[C][C]$ Irreg. bags blags füst gleks kagd kagt kets pagt pard peks poks schagd stard

English

$C S+B R+$. hangst kazz mact phadge phamb phangst phants plact sangst slangs slazz stract stramb tamb tazz tradge trazz zants

$C S+B R$-. clatt hald halse kalk kalse kalt phalk phaltz slaltz strald stralk stralse straltz tald taltz tralse tralt tratt

$C S-B R+$. quadge quamb quangst quapse quazz squact squazz swact swangst swants swazz twadge twangst twants twazz wact wamb wangst

$C S-B R$-. qualk qualse qualtz squald squalk squalse squaltz swalk swaltz twald twalk twalse twalt twaltz wald walse walt whald 


\section{Appendix B: Implementing the fitting process in $\mathbf{R}$}

While fitting the models described in the text has a certain flavour of regression to it, there are some important differences. Most critically are the two constraints that we have placed on the parameters: $\beta_{j} \in[0,1]$, and $\Sigma \beta_{j}=1$. Considerable work has been done to develop and implement estimation methods for models with inequality constraints such as $\beta_{j} \in[0,1]$ (Grömping, 2010). However, we know of no such work that has solved the problems presented by the $\Sigma \beta_{j}=1$ constraint. To address this problem, we turned to the optim function that is part of the base statistical analysis package in $\mathrm{R}$ ( $\mathrm{R}$ Core Team, 2013). optim is a very general optimisation package that allows the user to minimise any specified function, while also placing bounds on the returned values. That is, we can define a function, place upper and lower bounds on the returned weights, and optim will efficiently search the allowed parameter space to minimise our function. To satisfy (2), we defined the minimising function to be the residual sum of squares, and restricted the $\beta$ weights to fall between 0 and 1 . This ensures that $\hat{\beta}_{j} \in[0,1]$ is satisfied.

In all of the optimisation analyses, we used the following command in R:

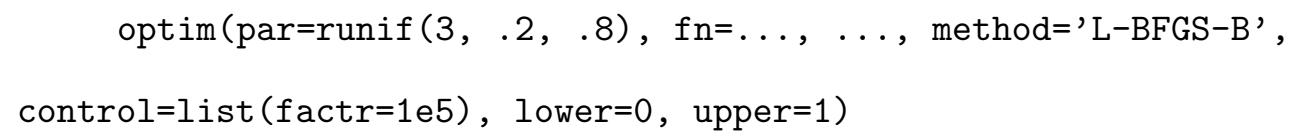

The parameters for optim operate as follows: "par=runif $(3, .2, .8)$ " initialises the $\beta_{i}$ s to random values between .2 and .8. " $\mathrm{fn}=\ldots, \ldots$ " specifies the function to be minimised along with any parameters it requires. In our case we used a simple function that calculates the residual sum of squares. "method=' $L-B F G S-B$ '" instructs optim to use an optimisation algorithm that allows for upper and lower bounds on the returned values (Byrd, Lu, Nocedal, \& Zhu, 1995). "factr=1e5" sets the convergence tolerance, and "lower $=0$, upper 1 " set the bounds on the returned 
values.

$$
\Sigma \beta_{j}=1 \text { Constraint. There is no way to explicitly tell optim to meet the }
$$
constraint that the $\beta$ s must sum to $1\left(\Sigma \beta_{j}=1\right)$. One way to ensure that the constraint is met is to simply scale the weights returned by optim using the formula:

$$
\beta_{j}^{\prime}=\beta_{j} / \Sigma \beta_{j}
$$

where $\beta_{j}^{\prime}$ are the new scaled weights, and are guaranteed to sum to 1 . However, since this adjustment follows the optimisation process, there is little reason to believe that the resulting $\beta_{j}^{\prime}$ s would remain an optimal solution to (2).

An alternative to simply scaling the $\beta_{j}$ s, is to make use of the influence of outliers on parameter estimation. For example, according to (2) optim is trying to satisfy the following 180 equations (two per item) simultaneously, by minimising the residual sum of squares (while also meeting the $\beta_{j} \in[0,1]$ constraint):

$$
\begin{gathered}
P_{1}(\text { Short })=\beta_{\text {gpc }} \times G P C_{\text {short }, 1}+\beta_{\text {csc }} \times C S C_{\text {short }, 1}+\beta_{b r c} \times B R C_{\text {short }, 1} \\
P_{1}(\text { Long })=\beta_{\text {gpc }} \times G P C_{\text {long }, 1}+\beta_{c s c} \times C S C_{\text {long }, 1}+\beta_{b r c} \times B R C_{\text {long }, 1} \\
\cdots \\
P_{90}(\text { Short })=\beta_{\text {gpc }} \times G P C_{\text {short }, 90}+\beta_{c s c} \times C S C_{\text {short }, 90}+\beta_{\text {brc }} \times B R C_{\text {short }, 90} \\
P_{90}(\text { Long })=\beta_{\text {gpc }} \times G P C_{\text {long }, 90}+\beta_{\text {csc }} \times C S C_{\text {long }, 90}+\beta_{\text {brc }} \times B R C_{\text {long }, 90}
\end{gathered}
$$

The introduction of a new data point that can only be met by satisfying the constraint that the $\Sigma \beta_{j}=1$ will put some pressure on optim to select appropriate parameters. For example,

$$
1=\beta_{g p c} \times 1+\beta_{c s c} \times 1+\beta_{b r c} \times 1
$$

(7) is equivalent to creating an artificial data point where all of the dependent and independent variables $[\mathrm{P}$ (Short), GPC, CSC, and BRC] are set to 1. Though (7) 
provides some pressure to satisfy $\Sigma \hat{\beta}_{j}=1$, it is unlikely to have a very large influence since it is only a single equation with roughly equal weight to the other 180 . However, dramatically increasing the weight of this data point will exert a much stronger influence on the final parameter selection. For example,

$$
10000=\beta_{g p c} \times 10000+\beta_{c s c} \times 10000+\beta_{b r c} \times 10000
$$

would put enormous pressure on optim to arrive at a set of weights that satisfy

$\Sigma \hat{\beta}_{j}=1$ without putting any further constraints on how the weights are apportioned to the strategies. Though (8) does not guarantee $\Sigma \hat{\beta}_{j}=1$ precisely, it is sufficiently strong for the present purposes. Other applications may require a larger multiplier.

Finally, because the number of items is not equal across all conditions in our studies, the sums of squares were weighted by item to ensure each condition contributed equally. For example in Experiment 1, items in the $\mathrm{V}[\mathrm{C}]$ Irregular and $\mathrm{V}[\mathrm{C}][\mathrm{C}]$ Irregular conditions received relatively more weight than items in the $\mathrm{V}[\mathrm{C}]$ Regular and $\mathrm{V}[\mathrm{C}][\mathrm{C}]$ Regular conditions. If this isn't done, there is a tendency for the Regular items to have a stronger influence on the eventual parameters. The weights applied to each item were determined as follows:

$$
\omega_{\text {type }}=\frac{.25}{n_{\text {type }}}
$$

where type is one of the four item types (e.g., V[C][C] Irregular in Experiment 1), $\omega_{\text {type }}$ is the weight assigned to items of that type, and $n_{\text {type }}$ is the total number of items of that type. As this formula implies, each item contributes equally to the influence of its category, but items in smaller categories have more influence than items in larger categories. These weights are then used in the usual weighted sum of squares formula 
Sublexical Correspondences 53

that optim is trying to minimise:

$$
S S_{\text {resid }}=\sum_{i}\left(\hat{Y}_{i}-\bar{Y}_{i}\right)^{2} \omega_{\text {type }_{i}}
$$




\section{Author Note}

We are grateful to Angela Heine and Tila Brink for collecting the Berlin data for Experiment 1B. We also thank Petra Schienmann and Reinhold Kliegl for their help with organising data collection at Potsdam University for Experiment 1B. We thank Johannes Ziegler for providing a list of German consistent words. Further thanks are due to Stephen Lupker, James Adelman, and three anonymous reviewers for their helpful comments on earlier versions of this paper.

This article was written as part of XS's doctoral dissertation under supervision of EM, AC, and MC. SR conducted the data analyses and contributed to the write-up and revision of the manuscript, and SP scored the English data.

Correspondence concerning this article should be addressed to Xenia Schmalz, Department of Cognitive Science, Macquarie University. Email: xenia.schmalz@mq.edu.au; phone: +61 (0)2 9850 2992; fax: +61 (0)2 98506059 


\section{Footnotes}

${ }^{1}$ It is not always true that graphemes are smaller (i.e., contain fewer letters than) bodies, e.g., the grapheme "igh" is larger than the body of the word "cat" ("-at"). For the sake of clarity, we follow the terminology of Ziegler and Goswami (2005) and refer to graphemes as small units, and bodies as large units.

${ }^{2}$ There are some differences associated with dialects. Here, we use the pronunciations given by the DRC's vocabulary and the Macquarie Essential Dictionary (5th Edition) as representative of Australian English, and the IPA as illustrated by Cox and Palethorpe (2007).

${ }^{3}$ Though we refer to the reliance on different types of correspondences as a "strategy", we do not mean to imply that readers consciously choose the type of correspondence that maximises the chance of correctly reading an unfamiliar word.

${ }^{4}$ In standard linear regression, only one of these two formulae would be required, since they are entirely dependent (i.e., $P_{i}($ Long $)=1-P_{i}($ Short $)$, etc...). In traditional regression, the only difference between the first and second equations would be the location of the estimated intercept and the sign of the slope. However, by removing the intercept term, our modelling strategy undermines this interdependence. Since the intercept is not free to vary (it is forced to be 0 ) the parameter estimates for $\mathrm{P}$ (short) would not match those for $\mathrm{P}$ (long). As a result, we must simultaneously fit both vowel pronunciations. While it is useful to use the language of regression to describe some of the procedures, it is very important to remember that the $\beta$ s here do not represent regression slopes, but weights. Also, if this were a regression problem, it would be more properly treated as a logistic regression problem. However, this would be incompatible with our interpretation of the weights as "the probability that a certain strategy is adopted." 
${ }^{5}$ It is noteworthy that Perry, Ziegler, Braun, and Zorzi (2010) report data with a similar set of nonwords to the current study (though the study was conducted with different aims): the authors manipulated the number of consonants in the coda, but rather than controlling for the consistency of the base-word, their nonwords differed in terms of the existence of the body in real words: the body either occurred in real German words, or it did not. In other words, they did not independently manipulate the predictions of body-rime correspondences and context-sensitive correspondences, and predictions of super-rules and body analogy were heavily correlated, $r(39)=0.78, p<0.001$, as were the predictions of super-rules and GPCs, $r(39)=0.51, p<0.001$. This means that the Perry et al. data is unsuitable for our purposes: the analysis would be unreliable, as it is impossible to disentangle reliance on bodies versus super-rules, and super-rules versus GPCs. 
Table 1

Percentage of Short Vowel Responses for Each Condition in Experiment 1, and the average predictions from each of the three types of correspondences

\begin{tabular}{ccccc}
\hline \hline Responses & $\mathrm{V}[\mathrm{C}]$ Regular & $\mathrm{V}[\mathrm{C}][\mathrm{C}]$ Regular & $\mathrm{V}[\mathrm{C}]$ Irregular & $\mathrm{V}[\mathrm{C}][\mathrm{C}]$ Irregular \\
\hline Example & "Wal" $\rightarrow$ "bral" & "Wald" $\rightarrow$ "brald" & "Bus" $\rightarrow$ "brus" & "Magd" $\rightarrow$ "bragd" \\
\% Short 1A & 47.25 & 83.69 & 84.63 & 61.04 \\
\% Short 1B & 37.28 & 86.79 & 72.95 & 62.84 \\
Correspondence Predictions & & & & \\
P(Short | GPC) & 70.21 & 79.53 & 90.59 & 78.77 \\
P(Short | CSC) & 26.2 & 92.57 & 62.82 & 91.38 \\
P(Short | BRC) & 2.76 & 100.00 & 100.00 & 0.00 \\
Model Predictions & & & & 60.53 \\
\% Short 1A & 44.68 & 87.04 & 87.83 & 61.70 \\
\% Short 1B & 36.58 & 89.78 & 83.67 & \\
\hline
\end{tabular}

Note: $\mathrm{GPC}=$ context-insensitive GPC $; \mathrm{CSC}=$ Context-sensitive correspondences $; \mathrm{BRC}=$

Body-rime correspondence 
Sublexical Correspondences 58

Table 2

Weightings for the three types of correspondences in Experiments $1 A, 1 B, 2 A$ and $2 B$

\begin{tabular}{ccccc}
\hline Correspondence type & 1A (German bil.) & 1B (German mon.) & 2A (English mon.) & 2B (English bil.) \\
\hline GPC & 0.56 & 0.38 & 0.05 & 0.03 \\
CSC & 0.19 & 0.35 & 0.69 & 0.61 \\
BRC & 0.26 & 0.27 & 0.26 & 0.36 \\
\hline
\end{tabular}


Table 3

Summary of the fits between the models and the observed response proportions. Each value is the correlation between the predictions from the GPC, CSC, BRC or model and the observed response pattern

\begin{tabular}{ccccc}
\hline \hline Sample & GPC & CSC & BRC & Optimal (95\%CI) \\
\hline 1A (German bil.) & .714 & .681 & .540 & $.844(.830, .847)$ \\
1B (German mon.) & .578 & .730 & .659 & $.827(.812, .832)$ \\
2A (English mon.) & .522 & .630 & .385 & $.729(.719, .731)$ \\
2B (English bil.) & .514 & .573 & .568 & $.792(.785, .793)$ \\
\hline
\end{tabular}


Table 4

Summary of vowel responses of English monolinguals (2A) and German/English bilinguals (2B), predictions from the three types of correspondences (context-independent GPCs; context-sensitive correspondences; body rhyme correspondences), and predictions from the model using the weights in Table 2.

\begin{tabular}{|c|c|c|c|c|c|}
\hline Experiment & Responses & CS-BR- & $\mathrm{CS}+\mathrm{BR}+$ & $\mathrm{CS}-\mathrm{BR}+$ & $\mathrm{CS}+\mathrm{BR}-$ \\
\hline Example & & "qualk" & "hangst" & "quadge" & "hald" \\
\hline \multicolumn{6}{|l|}{ Participant Responses } \\
\hline \multirow[t]{3}{*}{ 2A - Monolinguals } & $\% æ$ & 8.12 & 96.20 & 76.04 & 39.18 \\
\hline & $\%$ & 60.25 & 0.00 & 17.19 & 27.19 \\
\hline & $\% 0:$ & 10.63 & 0.00 & 0.88 & 8.77 \\
\hline \multirow[t]{3}{*}{ 2B - Bilinguals } & $\% æ$ & 8.07 & 83.33 & 62.50 & 41.20 \\
\hline & $\%$ & 38.24 & 0.93 & 19.91 & 27.31 \\
\hline & $\%$ & 52.19 & 0.00 & 0.00 & 7.87 \\
\hline
\end{tabular}

Correspondence Predictions

\begin{tabular}{|c|c|c|c|c|c|}
\hline \multirow[t]{3}{*}{ GPC } & $\mathrm{P}(\circledast \mid G P C)$ & 72.00 & 72.00 & 72.00 & 72.00 \\
\hline & $\mathrm{P}($ о $\mid G P C)$ & 5.00 & 5.00 & 5.00 & 5.00 \\
\hline & $\mathrm{P}(\mathrm{o:} \mid G P C)$ & 6.00 & 6.00 & 6.00 & 6.00 \\
\hline \multirow[t]{3}{*}{$\mathrm{CSC}$} & $\mathrm{P}(æ \mid C S C)$ & 29.00 & 77.00 & 29.00 & 77.00 \\
\hline & $\mathrm{P}($ о $\mid C S C)$ & 47.00 & 0.00 & 47.00 & 0.00 \\
\hline & $\mathrm{P}(\mathrm{o}: \mid C S C)$ & 0.00 & 0.00 & 0.00 & 0.00 \\
\hline \multirow[t]{3}{*}{ BRC } & $\mathrm{P}(\circledast \mid B R C)$ & 0.00 & 100.00 & 100.00 & 0.00 \\
\hline & $\mathrm{P}($ э $\mid B R C)$ & 0.00 & 0.00 & 0.00 & 0.00 \\
\hline & $\mathrm{P}(\mathrm{o}: \mid B R C)$ & 100.00 & 0.00 & 0.00 & 100.00 \\
\hline
\end{tabular}

Model Predictions

\begin{tabular}{cccccc} 
2A & $\% æ$ & 22.46 & 82.98 & 49.07 & 56.37 \\
& $\%$ \% & 33.35 & 0.14 & 33.35 & 0.14 \\
& $\%$ o: & 26.77 & 0.16 & 0.16 & 26.77 \\
& $\% æ$ & 18.88 & 85.34 & 55.39 & 48.84 \\
& $\%$ \% & 29.38 & 0.05 & 29.38 & 0.05 \\
& $\%$ о: & 36.57 & 0.07 & 0.07 & 36.57 \\
\hline
\end{tabular}


Table 5

Percentages of "Regular" responses (/æ/ in English, short vowels in German) given by the $D R C$ and $C D P+/ C D P++$

\begin{tabular}{ccccc}
\hline Model & CS+BR+ & CS-BR+ & CS+BR- & CS-BR- \\
\hline English Behavioural Data & 100 & 81 & 51 & 18 \\
English DRC Simulation 1 & 100 & 100 & 100 & 100 \\
English DRC Simulation 2 & 100 & 67 & 11 & 0 \\
English CDP + & 100 & 35 & 57 & 0 \\
English CDP++. & 100 & 43 & 44 & 0 \\
German Behavioural Data & 86 & 73 & 63 & 37 \\
German DRC & 100 & 0 & 100 & 0 \\
German CDP+ & 93 & 94 & 8 & 24 \\
\hline
\end{tabular}

